Systematic Review

\title{
A Systematic Review of the Effects of Physical Activity on Specific Academic Skills of School Students
}

\author{
Irineu Loturco*, Natalia P. Montoya, Marina B. Ferraz, Vanderson Berbat and Lucas A. Pereira
}

Citation: Loturco, I.; Montoya, N.P.; Ferraz, M.B.; Berbat, V.; Pereira, L.A. A Systematic Review of the Effects of Physical Activity on Specific Academic Skills of School Students. Educ. Sci. 2022, 12, 134. https:// doi.org/10.3390/educsci12020134

Academic Editor: Stephen A. Butterfield

Received: 31 December 2021

Accepted: 25 January 2022

Published: 18 February 2022

Publisher's Note: MDPI stays neutral with regard to jurisdictional claims in published maps and institutional affiliations.

Copyright: (C) 2022 by the authors. Licensee MDPI, Basel, Switzerland. This article is an open access article distributed under the terms and conditions of the Creative Commons Attribution (CC BY) license (https:// creativecommons.org/licenses/by/ $4.0 /)$.
Península Institute, São Paulo 01452-000, Brazil; natalia.puentes@institutopeninsula.org.br (N.P.M.); marina.ferraz@institutopeninsula.org.br (M.B.F.); vanderson.berbat@institutopeninsula.org.br (V.B.); lucas.pereira@narsp.com.br (L.A.P.)

* Correspondence: irineu.loturco@terra.com.br; Tel.: +55-11-3702-5284

\begin{abstract}
This systematic review examined the effects of distinct physical activity interventions on the academic achievement of school students based on an analysis of four distinct outcomes: mathematics, language, reading, and composite scores. This study was performed in accordance with the PRISMA guidelines and the QUORUM statement. A literature search was conducted using the PubMed-MEDLINE, Scopus, and Web of Science databases. Peer-reviewed studies published in English, Portuguese, and Spanish were considered. A random-effect meta-analysis was employed to determine the effect of interventions on academic performance. The effects between interventions and control groups were expressed as standardized mean differences. Thirty-one studies were included in the meta-analysis based on the inclusion and exclusion criteria. The exercise programs were not capable of significantly improving language, reading skills, and composite scores. Conversely, performance in math tests increased significantly after the interventions compared with the control groups. Regarding the overall effect, a significant improvement in academic achievement was detected after physical activity programs compared with controls. In conclusion, the positive effects of school-based physical education on academic performance are not uniform and may be higher for math skills. The implementation of evidence-based exercise programs in school settings emerges as a promising strategy to increase overall academic achievement in school-aged students.
\end{abstract}

Keywords: academic skills; active breaks; active classes; school grades; schoolchildren

\section{Introduction}

Over the last few decades, the rate of sedentarism has increased dramatically around the world, currently being considered a "public health issue" [1-4]. Overall, according to the World Health Organization (WHO), approximately one-third of individuals aged 15 years or older are physically inactive, which potentially results in more than 3 million premature or preventable deaths annually $[1,5,6]$. In fact, about $5.5 \%$ of all deaths worldwide have been attributed to sedentarism, a number that places this growing health concern among the five major risk factors for mortality in adult life [5,7]. Among other things, physical inactivity significantly increases the risk of several chronic diseases, such as different forms of cancer, diabetes, hypertension, dementia, coronary and cerebrovascular events, bone disorders, and obesity [8,9]. Together, these findings highlight the severity of this "global epidemic" and reinforce the need for urgent and effective strategies to increase the levels of physical activity and to reduce sedentary behavior among the general population $[3,10,11]$.

The adverse effects of sedentarism have been consistently recognized to affect distinct age groups, but more recently, a growing body of research has been conducted to evaluate its specific effects on youths [11-13]. Indeed, a progressive decline in physical activity participation is commonly observed during the transition from childhood to adolescence, suggesting that many adolescents are at risk of becoming sedentary adults [14-16]. It has been shown, for example, that a gradual decrease in physical fitness from childhood to adult life is associated with obesity and insulin resistance in adulthood [17]. From an applied 
standpoint, this means that the vast majority of children with obesity tend to become obese and "potentially unhealthy" adults $[17,18]$. In contrast, long-term programs and strategies aimed at maintaining high levels of physical activity during different life stages, from childhood to adulthood, may likely reduce the probability of developing obesity and other chronic diseases (e.g., type 2 diabetes, cardiovascular diseases, hypertension, osteoporosis, etc.) throughout adulthood [18-20]. As such, there is no doubt that regular exercise, especially when performed uninterruptedly from earlier life stages, has numerous physical and physiological benefits [21-24].

Lower levels of physical activity in childhood and adolescence are also associated with body image dissatisfaction, obesity, and adult depression [25-27]. On the other hand, the involvement in sport activities seems to influence the development of important coping and stress management skills in youths, which emphasizes the role played by physical activity in promoting mental health and in preventing mental illness [24,28-30]. Furthermore, there is evidence suggesting that physical activity can also improve cognitive function and learning abilities in both children and adolescents, thus impacting their academic achievement in different areas of knowledge (e.g., mathematics and languages). A recent review addressed this issue in a very comprehensive manner, finding meaningful relationships among physical activity, fitness levels, and academic performance in elementary-aged children (i.e., aged 5-13 years) [31]. Two other reviews have specifically examined the effectiveness of school-based physical activity in academic life, providing encouraging and relevant results [32,33]. Briefly put, the regular practice of physical education in school settings was shown to be capable of improving learning skills through different cognitive processes (e.g., increasing attention and concentration) and was equally efficient in inducing positive changes on a variety of important outcomes, such as quality of life, and social and affective behaviors $[32,33]$.

Although the benefits of physical education are well-established, to date, no review has systematically assessed its effects on different subjects and fields of study in a separate manner (i.e., analyzing its effects on mathematics, language, and reading skills) or in combination (i.e., using "composite scores"). This is essential to provide a deeper and more detailed understanding of the actual effects of physical education on the academic performance of school students. Therefore, the objective of this systematic review and meta-analysis was to evaluate the effects of distinct physical activity interventions on the academic achievement of school students, based on the individual analysis of four distinct outcomes: mathematics, language, reading, and composite scores. We hypothesized that the regular practice of physical education in schools would positively and equally affect academic performance, regardless of the subject being examined.

\section{Methods}

\subsection{Literature Search and Data Resources}

This research was completed in accordance with the Preferred Reported Items for Systematic Reviews (PRISMA) guidelines [34] and the QUORUM statement [35]. The literature search included studies published until 13 December 2021 and was conducted using the following databases: PubMed MEDLINE, Scopus, and Web of Science. Keywords were defined based on previous works $[36,37]$ and according to the study purpose by two authors (I.L. and L.A.P.). The following keywords were used in conjunction with the Boolean operators "AND" and "OR" as part of the search strategy: "students", "schoolchildren", "physical activity", "physical education", "sport", "exercise", "academic performance", "academic achievement", and "school grades". The reference lists from relevant articles were also examined to find any other potentially eligible studies.

\subsection{Eligibility Criteria}

Peer-reviewed studies published in English, Spanish, or Portuguese were considered for inclusion, and no sex or time of intervention restrictions were imposed. Studies were included based on the following criteria: (1) original studies; (2) quantitative assessment of 
"academic performance" pre- and post-intervention; (3) at least one group allocated to a physical activity intervention; and (4) samples composed of kindergarten, elementary, middle, or high-school students. Additionally, in relation to the exclusion criteria, studies were not considered for analysis if (1) they were written in other languages; (2) no comparison group was tested; (3) only acute effects were assessed; (4) they were qualitative studies; or (5) no full text or data regarding the main outcomes were available.

\subsection{Study Selection}

The initial search was carried out by two researchers (I.L. and L.A.P.). After the removal of duplicates, titles and abstracts were screened and studies not meeting the eligibility criteria were excluded. Subsequently, full texts of the remaining articles were analyzed. Next, in a blind, independent fashion, two reviewers selected the studies for inclusion (I.L. and L.A.P.), following the eligibility criteria. If no agreement was obtained, a third independent and experienced researcher, not involved in the study, was consulted.

\subsection{Data Extraction}

Mean, standard deviation (SD), and sample size data were extracted from the included manuscripts by two authors (I.L. and L.A.P.). If the descriptive data required were not presented in the articles, authors were contacted via email, and in case of no answer or if the requested data were not available, the study was not considered in the analysis. Any disagreements during the process of data extraction and analysis were resolved by consensus among two authors (I.L. and L.A.P.).

\subsection{Data Analysis}

The meta-analysis was performed using Review Manager software (RevMan 5.4.1; Cochrane Collaboration, Oxford, UK). A random-effect meta-analysis was conducted to determine the summary effect of the interventions on academic performance. Effects between intervention and control groups are expressed as standardized mean differences (SMD) and their 95\% confidence intervals (CI). Standardized mean differences were used because academic performance was obtained through distinct methods. Academic performance data were divided into subgroups, according to distinct assessed subjects (e.g., language, mathematics, reading, and composite scores).

Heterogeneity among studies was assessed using $I^{2}$ statistics. $I$ values ranged between $0 \%$ and $100 \%$ and were considered low, modest, or high for $<25 \%,>25-<50 \%$, and $>50 \%$, respectively. Heterogeneity may be assumed when the $p$ value of the $I$ test was $<0.05$ [38] Statistical significance was considered for $p<0.05$.

\subsection{Risk of Bias and Quality of the Studies}

Methodological quality and risk of bias were assessed through the Cochrane risk of bias tool (RoB 2.0) [39] by two authors independently (I.L. and L.A.P.), with disagreements being resolved by a third independent evaluator (not involved in the study), in accordance with the Cochrane Collaboration Guidelines [40].

\section{Results}

\subsection{Study Selection}

Figure 1 depicts a flow diagram of the process of study selection. A total of 2718 records were identified through database searching, and 16 additional studies were identified through other sources. After title and abstract screening, from the 1981 studies that remained after removal of duplicates, 1782 studies were excluded. As a result, 199 studies were assessed for eligibility and 31 studies were included in the meta-analysis based on the inclusion and exclusion criteria. From the articles included, 4 assessed academic performance through language tests [41-44], 24 used mathematics tests [41,42,44-65], 10 reading assessments $[44,46,47,49,50,57,59,60,63,64]$, and 9 evaluated academic achievement using composite scores (e.g., student's grades or standard tests) [41,44,59,66-71]. 


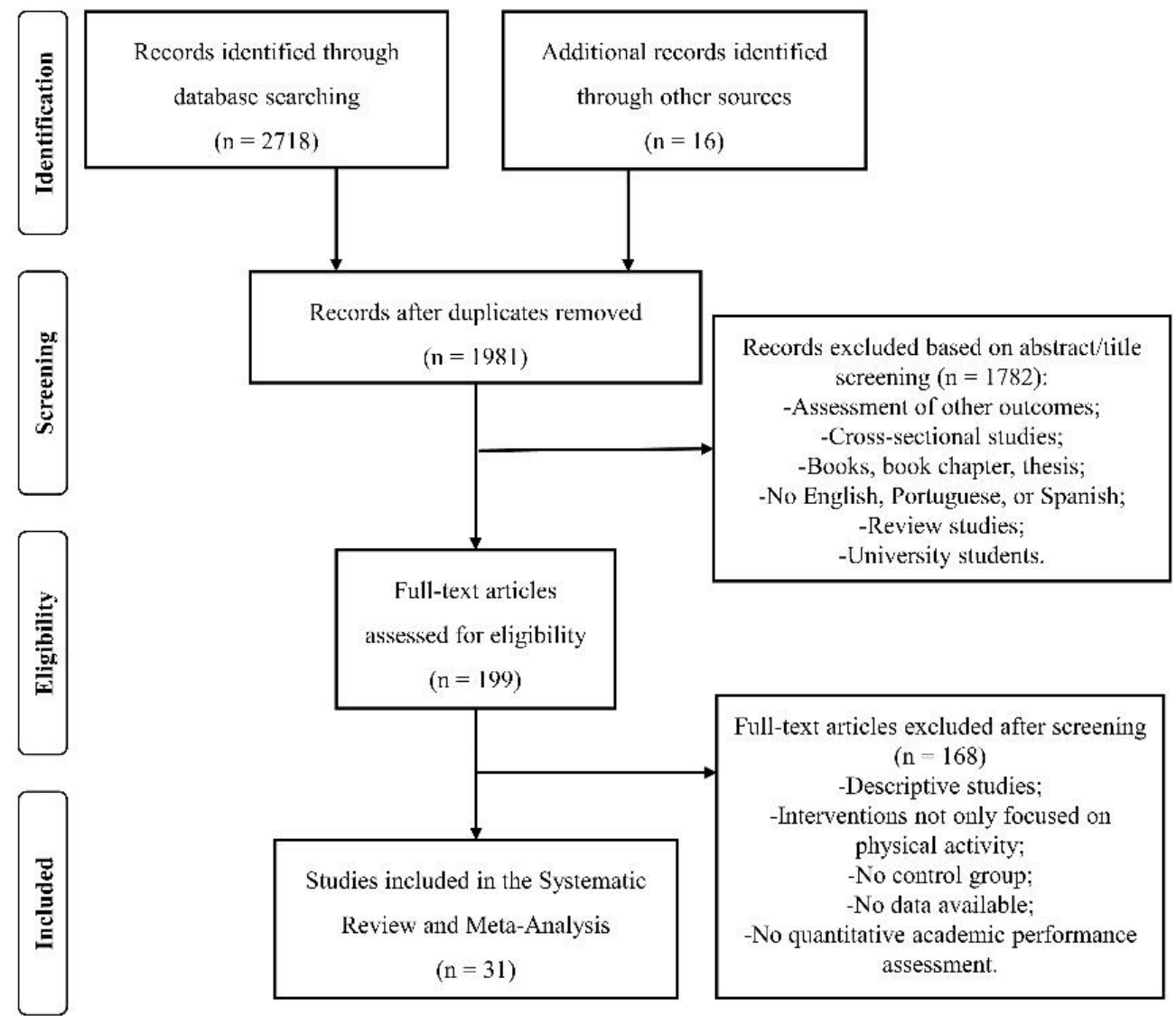

Figure 1. Flow diagram of the process of study selection.

\subsection{Characteristics of the Interventions}

The characteristics of the training programs and the overall risk of bias of the included studies are displayed in Table 1. The interventions implemented in the distinct studies comprised increasing the volume of physical education classes [41,68], applying classroombased activities in the regular school practices [66], promoting "active" breaks between regular classes [54,69], and employing specific programs of "active" lessons [49,51].

\subsection{Main Effects and Sub-Group Analyses}

Figure 2 depicts the forest plot for the effects of the physical activity interventions on the academic performance of students from several grades. The sub-group analyses revealed that the interventions were not capable of significantly improving academic achievement in language, reading, and composite scores. In contrast, performance in the mathematics tests significantly improved after the interventions when compared with control groups. In relation to the overall effect, a significant improvement in academic performance was noticed after physical activity interventions when compared with their respective controls. Overall, irrespective of the sub-group analyzed, the studies presented high levels of heterogeneity $\left(I^{2}>50 \%\right)$. 
Table 1. Characteristics of studies included in the meta-analysis.

\begin{tabular}{|c|c|c|c|c|c|c|}
\hline Study & Country & Participants & Intervention & Comparator & Outcome & $\begin{array}{c}\text { Overall Risk } \\
\text { of Bias }\end{array}$ \\
\hline $\begin{array}{c}\text { Ahmed et al. } \\
\text { (2007) }\end{array}$ & Canada & $\begin{array}{l}\text { Elementary school, } \\
9-11 \text { years, } C G=74 \\
\text { EG }=214\end{array}$ & $\begin{array}{l}\text { EG schools implemented classroom-based } \\
\text { activities for 15-min, } 5 \text { days/week. Activities } \\
\text { offered by teachers included skipping, dancing, } \\
\text { and resistance exercises performed with hand } \\
\text { grippers and exercise bands. }\end{array}$ & Regular PE $40 \mathrm{~min} /$ week & $\begin{array}{l}\text { Canadian Achievement Test (CAT-3; } \\
\text { a standardized test that evaluates } \\
\text { student skills related to specific } \\
\text { subject areas including mathematics, } \\
\text { reading, and language) }\end{array}$ & Some concerns \\
\hline $\begin{array}{l}\text { Ardoy et al. } \\
\qquad(2014)\end{array}$ & Spain & $\begin{array}{c}12-14 \text { years, } C G=18 \\
E G 1=26, E G 2=23\end{array}$ & $\begin{array}{c}\text { Adolescents in the EG1 had four PE } \\
\text { sessions / week, with the same contents and } \\
\text { pedagogical strategies as the sessions performed } \\
\text { by the CG. Adolescents in the EG2 complete four } \\
\text { PE sessions / week of high intensity, i.e., activities } \\
\text { with a heart rate }>120 \text { bpm. }\end{array}$ & $\begin{array}{l}\text { Usual PE sessions } \\
\text { according to the } \\
\text { National Education } \\
\text { Program in Spain, i.e., } \\
55 \text { min sessions twice } \\
\text { a week }\end{array}$ & $\begin{array}{l}\text { Academic achievement was } \\
\text { assessed using students' grades in } \\
\text { core subjects (mathematics and } \\
\text { language) and other subjects } \\
\text { (natural sciences, etc.). }\end{array}$ & Low risk \\
\hline $\begin{array}{l}\text { Beck et al. } \\
\text { (2016) }\end{array}$ & Denmark & $\begin{array}{l}165 \text { children, } \\
7.5 \text { years, } C G=57, \\
E G=55\end{array}$ & $\begin{array}{l}\text { Children in the EG performed inter-limb gross } \\
\text { motor-activities such as skipping, crawling, } \\
\text { throwing, and one-legged balancing. The } \\
\text { movements were performed while solving } \\
\text { mathematical questions (sessions of } \sim 60 \mathrm{~min} \text { ). }\end{array}$ & $\begin{array}{l}\text { CG employed } \\
\text { conventional } \\
\text { math teaching }\end{array}$ & $\begin{array}{l}\text { Mathematical achievement was } \\
\text { tested through a diagnostic test } \\
\text { developed by experts of the } \\
\text { neuropsychological field } \\
\text { in Denmark. }\end{array}$ & Some concerns \\
\hline $\begin{array}{l}\text { Donnelly et al. } \\
\text { (2017) }\end{array}$ & USA & $\begin{array}{c}\text { Grades } 2-3 \\
7-8 \text { years, } C G=316 \\
E G=316\end{array}$ & $\begin{array}{l}\text { Teachers were asked to deliver two } 10 \text { min } \\
\text { lessons/day }(\sim 4-5 \text { METs) in the subject of their } \\
\text { choice: one in the morning and one in the } \\
\text { afternoon, } 5 \text { days/week. }\end{array}$ & CG & $\begin{array}{l}\text { Academic achievement was } \\
\text { assessed via the Wechsler } \\
\text { Individual Achievement Test-Third } \\
\text { Edition (WIAT-III). }\end{array}$ & Low risk \\
\hline $\begin{array}{c}\text { Fakri and } \\
\text { Hashim (2020) }\end{array}$ & Malaysia & $\begin{array}{l}\text { Grade 5: } 10 \text { years, } \\
C G=35, E G=35\end{array}$ & $\begin{array}{l}\text { Participants in the EG engaged in physical activity } \\
\text { during math lessons. The activity comprises } \\
60 \text { min/week of moderate physically active lesson. }\end{array}$ & $\begin{array}{l}\text { The CG were taught via } \\
\text { traditional classroom } \\
\text { method. }\end{array}$ & $\begin{array}{l}\text { Academic achievement for } \\
\text { mathematic was measured using a } \\
\text { specific math test. }\end{array}$ & High risk \\
\hline
\end{tabular}


Table 1. Cont.

\begin{tabular}{|c|c|c|c|c|c|c|}
\hline Study & Country & Participants & Intervention & Comparator & Outcome & $\begin{array}{c}\text { Overall Risk } \\
\text { of Bias }\end{array}$ \\
\hline $\begin{array}{l}\text { Fedewa et al. } \\
\text { (2015) }\end{array}$ & USA & $\begin{array}{c}\text { Grades 3-5, } \\
C G=276, E G=153\end{array}$ & $\begin{array}{l}\text { The EG classrooms integrated physical activity } \\
\text { into their academic program for } 20 \mathrm{~min} / \text { day, } \\
5 \text { days / week using a set of movement cards. } \\
\text { These cards consisted of standardized } \\
\text { aerobic-based activities for } \sim 5 \text { min. }\end{array}$ & $\begin{array}{l}\text { The CG classrooms went } \\
\text { about business as usual, } \\
\text { with no additional } \\
\text { physical activity. }\end{array}$ & $\begin{array}{l}\text { Students' achievement in } \\
\text { mathematics and reading were } \\
\text { assessed with the "Measures of } \\
\text { Academic Progress" (MAP). }\end{array}$ & Some concerns \\
\hline $\begin{array}{l}\text { Gao et al. } \\
\text { (2013) }\end{array}$ & $\begin{array}{l}\text { USA- } \\
\text { Latino } \\
\text { School }\end{array}$ & $\begin{array}{c}\text { Grades } 3-5, \\
C G=58 \text { (year } 1) \text { and } \\
44 \text { (year } 2), E G=85 \\
\text { (year } 1) \text { and } \\
53(\text { year } 2)\end{array}$ & $\begin{array}{c}\text { Dance Revolution (DDR)_-15-min; center activity } \\
\text { station (e.g., aerobic } \\
\text { dance and jump rope)—15-min; 3/week. }\end{array}$ & CG & $\begin{array}{l}\text { To measure academic achievement, } \\
\text { reading and math scores for the } \\
\text { Utah Criterion-Referenced Test } \\
\text { were retrieved from the } \\
\text { school district. }\end{array}$ & High risk \\
\hline $\begin{array}{l}\text { Garcia- } \\
\text { Hermoso et al. } \\
\quad(2020)\end{array}$ & Chile & Grade 4: 8-10 years & $\begin{array}{l}\text { The intervention was delivered } 5 \text { times/week } \\
\text { before starting the first school-class. Active-Start is } \\
\text { mainly a program of cooperative physical games, } \\
\text { structured to make group cooperation essential to } \\
\text { game success and to encourage prosocial skills. }\end{array}$ & $\begin{array}{l}\text { Standard physical } \\
\text { education lessons }\end{array}$ & $\begin{array}{l}\text { Academic performance was } \\
\text { assessed using children's grades for } \\
\text { core subjects (mathematics } \\
\text { and language). }\end{array}$ & Low risk \\
\hline $\begin{array}{l}\text { Hagins and } \\
\text { Rundle (2016) }\end{array}$ & USA & $\begin{array}{c}\text { Grade } 9-11: \\
\sim 15 \text { years, } C G=48 \\
\mathrm{EG}=64\end{array}$ & $\begin{array}{l}\text { The PE class included weightlifting, stationary } \\
\text { biking, fitness exercises, and sports such as soccer } \\
\text { and volleyball. }\end{array}$ & $\begin{array}{l}\text { Yoga classes, } \\
\text { same volume }\end{array}$ & $\begin{array}{l}\text { Composite score was calculated as } \\
\text { the numeric average of scores of all } \\
\text { courses using a standard process } \\
\text { from New York City public schools. }\end{array}$ & Low risk \\
\hline $\begin{array}{l}\text { Hraste et al. } \\
\qquad(2018)\end{array}$ & Croatia & $\begin{array}{c}\text { Grade } 4: \\
10.4 \pm 0.55 \text { years, } \\
C G=17, E G=19\end{array}$ & $\begin{array}{l}\text { The EG classes consisted of four integrated lessons } \\
\text { of mathematics/geometry and PA. Each lesson } \\
\text { lasted for } 45 \mathrm{~min} \text {. }\end{array}$ & $\begin{array}{l}\text { CG: traditional teaching } \\
\text { methods for } \\
\text { mathematics }\end{array}$ & $\begin{array}{l}\text { The mathematics test was approved } \\
\text { by the National School Program as a } \\
\text { standard test for assessing } \\
\text { mathematical knowledge. }\end{array}$ & High risk \\
\hline $\begin{array}{l}\text { Layne et al. } \\
\quad(2020)\end{array}$ & USA & $\begin{array}{l}8-9 \text { years, } C G=21 \\
E G=19\end{array}$ & $\begin{array}{l}\text { EG played a FitNexx } 1.0 \text { active video game } \\
\text { 10-min/day before every mathematics class. }\end{array}$ & $\begin{array}{l}\text { CG waited in the } \\
\text { homeroom to enter the } \\
\text { mathematics class }\end{array}$ & $\begin{array}{l}\text { Mathematics tests were regular } \\
\text { assessments prepared by teachers. }\end{array}$ & Low risk \\
\hline $\begin{array}{l}\text { Lima et al. } \\
\quad(2020)\end{array}$ & Brazil & $\begin{array}{c}\sim 15 \text { years, } C G=188 \\
\mathrm{EG}=242\end{array}$ & $\begin{array}{l}\text { Schools that doubled the PE classes augmented } \\
\text { from } 2 \text { to } 4 \text { classes/week. }\end{array}$ & $\begin{array}{l}\text { Schools in the CG } \\
\text { maintained their } \\
\text { habitual routine }\end{array}$ & $\begin{array}{l}\text { Academic performance was } \\
\text { assessed by two different } \\
\text { mathematics tests. }\end{array}$ & Low risk \\
\hline
\end{tabular}


Table 1. Cont.

\begin{tabular}{|c|c|c|c|c|c|c|}
\hline Study & Country & Participants & Intervention & Comparator & Outcome & $\begin{array}{c}\text { Overall Risk } \\
\text { of Bias }\end{array}$ \\
\hline $\begin{array}{l}\text { Lubans et al. } \\
\qquad(2018)\end{array}$ & Australia & $\begin{array}{l}\text { Grade 8: } \sim 13 \text { years, } \\
C G=580, E G=584\end{array}$ & $\begin{array}{c}\text { Activity and Motivation in Physical } \\
\text { Education (AMPED) }\end{array}$ & CG & $\begin{array}{l}\text { Mathematics performance was } \\
\text { assessed as part of the National } \\
\text { Assessment Program Literacy \& } \\
\text { Numeracy (NAPLAN). }\end{array}$ & Low risk \\
\hline $\begin{array}{l}\text { Mavilidi et al. } \\
\text { (2018a) }\end{array}$ & Australia & $\begin{array}{l}\text { Grade } 4: 10-11 \text { years, } \\
\qquad \mathrm{CG}=26, \mathrm{EG}=29\end{array}$ & $\begin{array}{l}\text { "Thinking While Moving in English", a primary } \\
\text { school program that integrates physical activity } \\
\text { into English lessons. }\end{array}$ & CG & $\begin{array}{l}\text { Children's spelling and grammar } \\
\text { skills were assessed using the South } \\
\text { Australian Spelling and Grammar } \\
\text { and Punctuation tests. }\end{array}$ & High risk \\
\hline $\begin{array}{l}\text { Mavilidi et al. } \\
\text { (2018b) }\end{array}$ & Australia & $\begin{array}{c}4.7 \pm 0.5 \text { years, } \\
\mathrm{CG}=27, \mathrm{EG}=30\end{array}$ & $\begin{array}{l}\text { In the EG, children performed physical activity } \\
\text { related to learning tasks. }\end{array}$ & CG & $\begin{array}{l}\text { Math test consisted of counting, } \\
\text { number line estimation, block } \\
\text { counting, and numerical magnitude } \\
\text { comparison and identification. }\end{array}$ & Some concerns \\
\hline $\begin{array}{l}\text { Mavilidi et al. } \\
\text { (2020) }\end{array}$ & Australia & $\begin{array}{l}\text { Grades } 3-4: \sim 9 \text { years, } \\
\qquad \mathrm{CG}=29, \mathrm{EG}=29\end{array}$ & $\begin{array}{l}\text { This EG involved two types of activity breaks } \\
\text { delivered in separate classes, totaling } 5 \text { min. }\end{array}$ & CG & $\begin{array}{l}\text { Students' mastery of basic facts was } \\
\text { assessed using the Stage } 2 \text { version } \\
\text { of the Individual Basic Facts } \\
\text { Assessment Tool (IBFA). }\end{array}$ & Low risk \\
\hline $\begin{array}{l}\text { Mead et al. } \\
\quad(2016)\end{array}$ & USA & $\begin{array}{l}\text { Grade 6: } 11-12 \text { years, } \\
\qquad G=22, E G=28\end{array}$ & $\begin{array}{l}\text { Five-minute physical activity breaks were } \\
\text { implemented and occurred immediately after the } \\
\text { math message but prior to the beginning of } \\
\text { work time. }\end{array}$ & CG & $\begin{array}{c}\text { Minnesota Comprehensive } \\
\text { Assessments (MCAs): state } \\
\text { achievement tests in mathematics, } \\
\text { reading, and science. Measures of } \\
\text { Academic Progress (MAP): } \\
\text { standardized test assessing reading, } \\
\text { mathematics, and language }\end{array}$ & Low risk \\
\hline $\begin{array}{l}\text { Mullender- } \\
\text { Wijnsma et al. } \\
\text { (2016) }\end{array}$ & $\begin{array}{l}\text { The } \\
\text { Nether- } \\
\text { lands }\end{array}$ & $\begin{array}{l}\text { Grades } 2-3: \sim 8 \text { years, } \\
\mathrm{CG}=170, \mathrm{EG}=181\end{array}$ & $\begin{array}{l}\text { The EG participated in Fit \& Vaardig op } \\
\text { School lessons. }\end{array}$ & CG & $\begin{array}{l}\text { Academic achievement was } \\
\text { measured by two mathematics and } \\
\text { two language tests. }\end{array}$ & Low risk \\
\hline
\end{tabular}


Table 1. Cont.

\begin{tabular}{|c|c|c|c|c|c|c|}
\hline Study & Country & Participants & Intervention & Comparator & Outcome & $\begin{array}{l}\text { Overall Risk } \\
\text { of Bias }\end{array}$ \\
\hline $\begin{array}{l}\text { Pinto-Escalona } \\
\text { and Martínez- } \\
\text { de-Quel } \\
\text { (2019) }\end{array}$ & Spain & $\begin{array}{l}13.6 \pm 0.7 \text { years, } \\
\mathrm{CG}=60, \mathrm{EG}=56\end{array}$ & $\begin{array}{l}\text { The experimental group participated in } 10 \text { min of } \\
\text { moderate to vigorous physical exercise related to } \\
\text { the contents of the teaching unit. }\end{array}$ & $\begin{array}{l}\text { The CG carried out } 10 \\
\text { min of study and review } \\
\text { seated in their chairs. }\end{array}$ & $\begin{array}{l}\text { An objective assessment test was } \\
\text { drawn up for the contents of } \\
\text { Spanish language and literature. }\end{array}$ & Low risk \\
\hline $\begin{array}{l}\text { Polo-Recuero } \\
\text { et al. (2020) }\end{array}$ & Spain & $\begin{array}{c}\sim 13 \text { years, } C G=13 \\
E G=14\end{array}$ & $\begin{array}{l}\text { EG used bike desks during their four weekly } \\
\text { math sessions. }\end{array}$ & CG & Mathematics test & Low risk \\
\hline $\begin{array}{c}\text { Quinto- } \\
\text { Romani and } \\
\text { Klausen (2016) }\end{array}$ & Denmark & $\begin{array}{l}\text { Grade 6: } 11-13 \text { years, } \\
\text { Grade 8: } 13-15 \text { years, } \\
C G=371, E G=554\end{array}$ & The core of the intervention was physical activity & CG & Grade point average & Some concerns \\
\hline $\begin{array}{l}\text { Resaland et al. } \\
\text { (2016) }\end{array}$ & Norway & $\begin{array}{c}\text { Grade } 5: \\
10.2 \pm 0.3 \text { years, } \\
C G=533, E G=596\end{array}$ & $\begin{array}{l}\text { (1) } 90 \mathrm{~min} \text { / week of physically active educational } \\
\text { lessons mainly carried out in the school } \\
\text { playground; (2) } 5 \text { min/day of physical activity } \\
\text { breaks during classroom lessons; (3) } 10 \text { min/day } \\
\text { physical activity homework }\end{array}$ & CG & $\begin{array}{l}\text { Academic performance in } \\
\text { numeracy, reading, and English was } \\
\text { measured via standardized } \\
\text { Norwegian national tests. }\end{array}$ & Low risk \\
\hline $\begin{array}{l}\text { Riley et al. } \\
\text { (2016) }\end{array}$ & Australia & $\begin{array}{l}11.1 \pm 0.73 \text { years, } \\
\mathrm{CG}=98, \mathrm{EG}=142\end{array}$ & $\begin{array}{l}\text { The program involved classroom teachers } \\
\text { adapting mathematics lessons over a } 6 \text { week } \\
(3 \times 60 \text { min sessions/week) period to ensure } \\
\text { students were involved in } \\
\text { movement-based learning. }\end{array}$ & CG & $\begin{array}{l}\text { Mathematics performance was } \\
\text { measured using a standardized } \\
\text { mathematics progressive } \\
\text { achievement test. }\end{array}$ & Low risk \\
\hline $\begin{array}{l}\text { Sallis et al. } \\
\text { (1999) }\end{array}$ & USA & $\begin{array}{c}\text { Grades } 4-6: \text { } ~ 9 \text { years, } \\
\text { CG1 = 124, } \\
\text { CG2 }=141 \\
\text { EG1 }=177, \text { EG2 }=312\end{array}$ & $\begin{array}{l}\text { SPARK is a comprehensive curriculum and } \\
\text { professional development program designed to } \\
\text { promote physical activity in/out of school. }\end{array}$ & CG & $\begin{array}{l}\text { Academic achievement was } \\
\text { measured using Metropolitan } \\
\text { Achievement Tests. }\end{array}$ & Low risk \\
\hline $\begin{array}{l}\text { Shore et al. } \\
\text { (2014) }\end{array}$ & USA & $\begin{array}{c}\text { Grade 6: } 11 \text { years, } \\
\mathrm{CG}=46, \mathrm{EG}=46\end{array}$ & $\begin{array}{c}\text { All students had PE class twice every } 6 \text { days. } \\
\text { Students in the EG also received two verbal } \\
\text { prompts in PE class to attain a } 3200 \text { step-count goal } \\
\text { during classes. }\end{array}$ & $\begin{array}{c}\text { The CG participated in } \\
\text { the standard } \\
\text { PE curriculum. }\end{array}$ & $\begin{array}{c}\text { Academic performance was } \\
\text { determined by grade } \\
\text { point averages. }\end{array}$ & Low risk \\
\hline $\begin{array}{l}\text { Shoval et al. } \\
\qquad(2018)\end{array}$ & Israel & $\begin{array}{l}4-6 \text { years, } C G=45 \\
\text { EG1 }=61, E G 2=54\end{array}$ & Two movement-based interventions & $\begin{array}{l}\text { Children learned in a } \\
\text { conventional kinder- } \\
\text { garten environment. }\end{array}$ & $\begin{array}{c}\text { Mathematics Achievement Test } \\
\text { (MAT) Comprehension Reading } \\
\text { Test (CRT) }\end{array}$ & High risk \\
\hline
\end{tabular}


Table 1. Cont.

\begin{tabular}{|c|c|c|c|c|c|c|}
\hline Study & Country & Participants & Intervention & Comparator & Outcome & $\begin{array}{c}\text { Overall Risk } \\
\text { of Bias }\end{array}$ \\
\hline $\begin{array}{l}\text { Tarp et al. } \\
(2016)\end{array}$ & Denmark & $\begin{array}{c}\text { Grades 6-7: } \\
\text { 12-14 years, } \\
\mathrm{CG}=419, \mathrm{EG}=191\end{array}$ & $\begin{array}{l}\text { Targeting classroom, recess, and leisure-time } \\
\text { activity and through active transportation }\end{array}$ & CG & $\begin{array}{l}\text { Custom-made grade-specific } \\
\text { mathematics tests were used to } \\
\text { assess academic performance. }\end{array}$ & Low risk \\
\hline $\begin{array}{l}\text { Vazou and } \\
\text { Skrade (2017) }\end{array}$ & USA & $\begin{array}{c}\text { Grades } 4-5, \\
\mathrm{CG}=118, \mathrm{EG}=107\end{array}$ & $\begin{array}{l}\text { The EG utilized a kit named "Move for Thought", } \\
\text { which offers flexible options for integrating PA } \\
\text { with academic subjects. }\end{array}$ & $\begin{array}{l}\text { CG: math content } \\
\text { without any changes in } \\
\text { their regular } \\
\text { instructional format }\end{array}$ & $\begin{array}{l}\text { Timed, comprehensive, grade-level } \\
\text { appropriate, and standardized math } \\
\text { tests in algebra and operations were } \\
\text { used to measure math performance. }\end{array}$ & High risk \\
\hline $\begin{array}{l}\text { Watson et al. } \\
\qquad(2019)\end{array}$ & Australia & $\begin{array}{c}\text { Grades } 3-4: \\
8-10 \text { years, } C G=218 \\
\text { EG }=123\end{array}$ & $\begin{array}{l}\text { EG involved teachers incorporating } 3 \times 5 \text { min } \\
\text { active breaks into their classroom routine daily. }\end{array}$ & CG & $\begin{array}{l}\text { Academic achievement was } \\
\text { assessed using } 1 \text { min tests in } \\
\text { reading and mathematics. }\end{array}$ & Low risk \\
\hline
\end{tabular}

CG: control group; EG: experimental group; PE: physical education. 


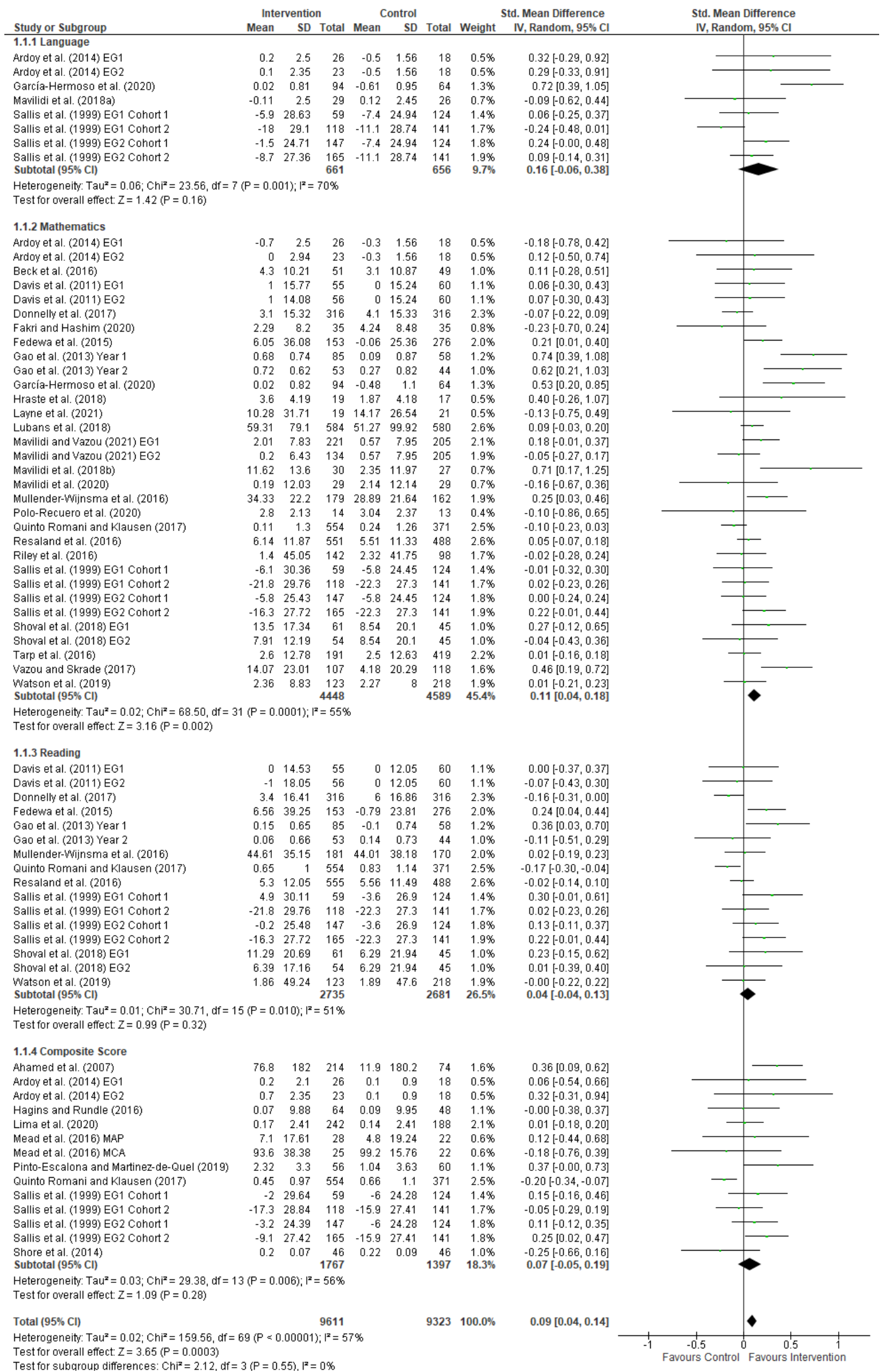

Figure 2. Standardized mean difference (95\% confidence intervals, CI) between intervention and control groups. Squares represent the SMD for each trial. Diamonds represent the pooled SMD across trials. 


\section{Discussion}

In this systematic review, we examined the effects of different types of physical activity programs on the academic performance of school students. After analyzing these effects on an individual basis (i.e., via sub-group analyses), it was possible to verify that these interventions did not increase academic achievement in language skills, reading skills, and composite scores. In contrast, performance in mathematics significantly improved, which reveals the important role played by physical education in some specific learning processes and cognitive functions. Moreover, an analysis of the "overall effect" (i.e., through the use of a pooled computation, considering all scores simultaneously) confirmed that physical activity interventions are useful resources for enhancing the academic performance of children and adolescents.

The contrasting results among the different subjects and learning abilities (i.e., mathematics, language, and reading skills, and composite scores) are difficult to interpret and may also be influenced by a variety of external factors (i.e., type and mode of exercise, duration and frequency of training intervention, etc.) [59]. However, some similar results can be found in several studies, thus requiring more detailed discussion. In this regard, Quinto Romani et al. [59] examined the effects of five distinct training programs on the academic performance of 1479 Danish students and reported some interesting findings: (1) on average, all interventions seem to have a "very limited beneficial impact" on academic achievement; (2) this beneficial impact tends to be higher for "high-intensity training programs". The superior effects of more intensive training sessions on cognition and learning skills were also highlighted in other investigations on this topic $[41,44,50,62]$. For example, Ardoy et al. [41] analyzed the role played by exercise frequency and intensity on academic success by comparing the effects of three different experimental conditions: (1) standard physical education ("control group") (i.e., sessions prescribed according to the "National Law of Education"; $2 \times 55 \mathrm{~min} /$ week); (2) increased exercise volume ("experimental group 1"; “EG1") (i.e., $4 \times 55 \mathrm{~min} /$ week); and (3) increased exercise volume and intensity ("EG2") (i.e., more intensive 55 min sessions; 4 times/week). In general, the EG2 group improved more than the control group and the EG1 group in several cognitive functions and domains (i.e., non-verbal and verbal skills, abstract reasoning, and spatial and numerical skills), which also indicates that the simple increase in frequency of physical education sessions (e.g., from 2 to 4 times/week) is not sufficient to promote positive effects on academic achievement (at least when these increases are not accompanied by concomitant changes in exercise intensity). With that said, teachers and policy makers should be aware that more intensive physical activities are required to achieve greater gains in academic performance.

The positive effects of school-based physical activity programs were larger for mathematics skills, with sub-group analyses revealing no significant effects for language- and reading-related abilities and composite scores (Figure 2). Accordingly, Mavilidi et al. [43] reported no improvements in cognitive function and some specific learning outcomes (i.e., grammar skills) after examining the effects of a 4-week school-based program that integrated physical activity into English lessons in children aged 11-12 years. Likewise, Shore et al. [70] did not find any differences in academic achievement (assessed by composite scores) after comparing the effects of a "school-based pedometer intervention" (i.e., a sample of sixth-grade students who received instructions to achieve 3200 steps during physical education classes and a total of 10,000 daily steps for 6 weeks) with the effects of "standard physical education" (i.e., students who participated in regular physical education classes). Despite the short duration of the abovementioned studies (i.e., $\leq 6$ weeks), their findings contrast with previous investigations with similar time periods and aims, which assessed the effects of physical activity on mathematics skills. For instance, a 6-week study comparing the effects of three different strategies (i.e., "conventional math teaching" versus math teaching combined with "fine" or "gross" motor tasks) showed that participation in the math classes that integrated more vigorous physical activities (i.e., inter-limb movements such as skipping, throwing, and one-legged balancing) could positively influence 
mathematical achievements in pre-adolescent children [45]. Vazou et al. [62] observed a similar trend in an 8-week intervention that tested the effects of physical activity (combined with math) in fourth- and fifth-grade students. In that study, the increase in math performance was significantly larger in the "integrated physical activity group" (compared with that of the control group, who participated in traditional math classes). From these results, it is possible to infer that (1) improvements in math skills are more expected and consistent than in other skills (e.g., improvements in language or reading abilities) after short- or mid-term ( $\leq 8$ weeks) physical education programs and (2) the integration of physical activity into regular classes (especially mathematics) could be an effective strategy to enhance learning in school-aged students.

In fact, the benefits of physical education for math learning appear to be more pronounced even in long-term studies. In this sense, Lubans et al. [53] demonstrated the positive (small-to-medium) effects of a 15-month "multicomponent physical activity program" (including physical education classes with moderate-to-vigorous exercises) on mathematics performance in an investigation conducted with hundreds of eighth-grade students. Mullender-Wijnsma et al. [57] confirmed this tendency after analyzing the results of a 2-year intervention that integrated physical activities into math and spelling lessons in almost $500 \mathrm{~s}$ - and third-grade students from 12 elementary schools. Curiously, in that study, both spelling and general math skills increased (compared with the control group); nevertheless, the positive effect on mathematics performance (revealed by the "child academic monitoring system test") was already detected after one year of intervention, whereas spelling ability was only enhanced after two years. Together, these findings suggest that the improvements in mathematics learning obtained through the implementation of systematic physical education programs might be superior, at least when compared with languageand reading-related abilities. This also holds true for analyses integrating a variety of learning scores (i.e., composite scores) and agrees with the main outcome of this systematic review, which highlights the positive influence of physical education programs on mathematics achievement.

\section{Conclusions}

In general, the positive effects of school-based physical education programs on academic performance are not uniform and seem to be higher for mathematics skills. These effects may be optimized with the integration of multi-component exercise schemes into regular classes of different education levels as an efficient and alternative learning strategy. The concomitant prescription of "gross motor tasks" and more intensive (and appropriate) physical activities is also recommended in an attempt to maximize students' learning outcomes. In addition to the recognized benefits of systematic exercise on both health and psychological well-being, the implementation of evidence-based physical education programs in distinct school settings emerges as a promising strategy to increase overall academic achievement in school-aged students.

Author Contributions: Conceptualization, I.L., N.P.M., M.B.F., V.B., and L.A.P.; methodology, I.L. and L.A.P.; formal analysis, I.L. and L.A.P.; investigation, I.L. and L.A.P.; data curation, I.L. and L.A.P.; writing—original draft preparation, I.L. and L.A.P.; writing—review and editing, I.L. and L.A.P.; visualization, I.L., N.P.M., M.B.F., V.B., and L.A.P.; supervision, I.L. and L.A.P.; project administration, I.L., N.P.M., M.B.F., V.B., and L.A.P.; All authors have read and agreed to the published version of the manuscript.

Funding: This research received no external funding.

Institutional Review Board Statement: Not applicable.

Informed Consent Statement: Not applicable.

Data Availability Statement: Not applicable.

Conflicts of Interest: The authors declare no conflict of interest. 


\section{References}

1. Baker, B.; Castelli, D. Physical activity and sedentary behavior influences on executive function in daily living. In Neuroergonomics; Chang, S.N., Ed.; Springer: Champaign, IL, USA, 2020; pp. 161-181.

2. Bermúdez, V.J.; Rojas, J.J.; Córdova, E.B.; Añez, R.; Toledo, A.; Aguirre, M.A.; Cano, C.; Arraiz, N.; Velasco, M.; López-Miranda, J. International physical activity questionnaire overestimation is ameliorated by individual analysis of the scores. Am. J. Ther. 2013, 20, 448-458. [CrossRef] [PubMed]

3. Di Cesare, M.; Sorić, M.; Bovet, P.; Miranda, J.J.; Bhutta, Z.; Stevens, G.A.; Laxmaiah, A.; Kengne, A.P.; Bentham, J. The epidemiological burden of obesity in childhood: A worldwide epidemic requiring urgent action. BMC Med. 2019, 17, 212. [CrossRef] [PubMed]

4. Middelbeek, L.; Breda, J. Obesity and sedentarism: Reviewing the current situation within the who european region. Curr. Obes. Rep. 2013, 2, 42-49. [CrossRef]

5. Mathers, C.; Stevens, G.; Mascarenhas, M. Global Health Risks: Mortality and Burden of Disease Attributable to Selected Major Risks; World Health Organization: Geneva, Switzerland, 2009.

6. $\quad$ Normansell, R.A.; Holmes, R.; Victor, C.R.; Cook, D.G.; Kerry, S.; Iliffe, S.; Ussher, M.; Ekelund, U.; Fox-Rushby, J.; Whincup, P. Op23 exploring the reasons for non-participation in physical activity interventions: Pace-up trial qualitative findings. J. Epidemiol. Community Health 2014, 68, A14. [CrossRef]

7. Sánchez Oliver, A.J.; Martín García, C.; Gálvez Ruiz, P.; González-Jurado, J.A. Mortality and economic expenses of cardiovascular diseases caused by physical inactivity in spain. J. Phys. Educ. Sport 2018, 18, 1420-1426.

8. Knight, J.A. Physical inactivity: Associated diseases and disorders. Ann. Clin. Lab. Sci. 2012, 42, 320-337.

9. World Health Organization. Global Status Report on Noncommunicable Diseases 2014; World Health Organization: Geneva, Switzerland, 2014.

10. Singh, R.; Pattisapu, A.; Emery, M.S. US physical activity guidelines: Current state, impact and future directions. Trends Cardiovasc. Med. 2020, 30, 407-412. [CrossRef]

11. Tran, B.X.; Dang, K.A.; Le, H.T.; Ha, G.H.; Nguyen, L.H.; Nguyen, T.H.; Tran, T.H.; Latkin, C.A.; Ho, C.S.H.; Ho, R.C.M. Global evolution of obesity research in children and youths: Setting priorities for interventions and policies. Obes. Facts 2019, 12, 137-149. [CrossRef]

12. Arancibia, B.A.V.; Silva, F.C.; Dos Santos, P.D.; Gutierres Filho, P.J.B.; Silva, R. Prevalence of physical inactivity among adolescents in brazil: Systematic review of observational studies. Phys. Educ. Sport 2015, 34, 331-358. [CrossRef]

13. Gómez, S.F.; Homs, C.; Wärnberg, J.; Medrano, M.; Gonzalez-Gross, M.; Gusi, N.; Aznar, S.; Cascales, E.M.; González-Valeiro, M.; Serra-Majem, L.; et al. Study protocol of a population-based cohort investigating physical activity, sedentarism, lifestyles and obesity in spanish youth: The pasos study. BMJ Open 2020, 10, e036210. [CrossRef]

14. Do Nascimento, A.C.S.; Suano-Souza, F.I.; Sarni, R.O.S. Extracurricular physical activities practiced by children: Relationship with parents' nutritional status and level of activity. Nutrire 2019, 44, 7. [CrossRef]

15. McCaul, K.; Baker, J.; Yardley, J.K. Predicting substance use from physical activity intensity in adolescents. Pediatr. Exerc. Sci. 2004, 16, 277-289. [CrossRef]

16. Pate, R.R.; Long, B.J.; Heath, G.W. Descriptive epidemiology of physical activity in adolescents. Pediatr. Exerc. Sci. 1994, 6, 434-447. [CrossRef]

17. Clarke, W.R.; Lauer, R.M. Does childhood obesity track into adulthood? Crit. Rev. Food Sci. Nutr. 1993, 33, 423-430. [CrossRef]

18. Dwyer, T.; Magnussen, C.G.; Schmidt, M.D.; Ukoumunne, O.C.; Ponsonby, A.L.; Raitakari, O.T.; Zimmet, P.Z.; Blair, S.N.; Thomson, R.; Cleland, V.J.; et al. Decline in physical fitness from childhood to adulthood associated with increased obesity and insulin resistance in adults. Diabetes Care 2009, 32, 683-687. [CrossRef] [PubMed]

19. Bouchard, C.; Després, J.P. Physical activity and health: Atherosclerotic, metabolic, and hypertensive diseases. Res. Q. Exerc. Sport 1995, 66, 268-275. [CrossRef]

20. Kravitz, L. The 25 most significant health benefits of physical activity and exercise. IDEA Fit. J. 2007, 4, 54-63.

21. Biddle, S.; Fox, K.R.; Boutcher, S.H. Physical Activity and Psychological Well-Being; Routledge: London, UK, $2000 ;$ Volume 552.

22. Calfas, K.J.; Taylor, W.C. Effects of physical activity on psychological variables in adolescents. Pediatr. Exerc. Sci. 1994, 6, 406-423. [CrossRef]

23. Kokkinos, P. Physical activity, health benefits, and mortality risk. ISRN Cardiol. 2012, 2012, 718789. [CrossRef]

24. Malina, R.M. Physical activity and fitness: Pathways from childhood to adulthood. Am. J. Hum. Biol. 2001, 13, 162-172. [CrossRef]

25. Delgado-Floody, P.; Guzmán-Guzmán, I.P.; Caamaño-Navarrete, F.; Jerez-Mayorga, D.; Zulic-Agramunt, C.; Cofré-Lizama, A Depression is associated with lower levels of physical activity, body image dissatisfaction, and obesity in chilean preadolescents. Psychol. Health Med. 2021, 26, 518-531. [CrossRef] [PubMed]

26. Jacka, F.N.; Pasco, J.A.; Williams, L.J.; Leslie, E.R.; Dodd, S.; Nicholson, G.C.; Kotowicz, M.A.; Berk, M. Lower levels of physical activity in childhood associated with adult depression. J. Sci. Med. Sport 2011, 14, 222-226. [CrossRef] [PubMed]

27. Tomson, L.M.; Pangrazi, R.P.; Friedman, G.; Hutchison, N. Childhood depressive symptoms, physical activity and health related fitness. J. Sport Exerc. Psychol. 2003, 25, 419-439. [CrossRef]

28. Brand, S.; Kalak, N.; Gerber, M.; Clough, P.J.; Lemola, S.; Sadeghi Bahmani, D.; Pühse, U.; Holsboer-Trachsler, E. During early to mid adolescence, moderate to vigorous physical activity is associated with restoring sleep, psychological functioning, mental toughness and male gender. J. Sports Sci. 2017, 35, 426-434. [CrossRef] [PubMed] 
29. Doré, I.; Sylvester, B.; Sabiston, C.; Sylvestre, M.P.; O'Loughlin, J.; Brunet, J.; Bélanger, M. Mechanisms underpinning the association between physical activity and mental health in adolescence: A 6-year study. Int. J. Behav. Nutr. Phys. Act. 2020, 17, 9. [CrossRef]

30. Slater, A.; Tiggemann, M. The contribution of physical activity and media use during childhood and adolescence to adult women's body image. J. Health Psychol. 2006, 11, 553-565. [CrossRef]

31. Donnelly, J.E.; Hillman, C.H.; Castelli, D.; Etnier, J.L.; Lee, S.; Tomporowski, P.; Lambourne, K.; Szabo-Reed, A.N. Physical activity, fitness, cognitive function, and academic achievement in children: A systematic review. Med. Sci. Sports Exerc. 2016, 48, 1223-1224. [CrossRef]

32. Marques, A.; Corrales, F.R.G.; Martins, J.; Catunda, R.; Sarmento, H. Association between physical education, school-based physical activity, and academic performance: A systematic review. New Trends Phys. Educ. Sport Recreat. 2017, 31, 316-320.

33. Zach, S.; Shoval, E.; Lidor, R. Physical education and academic achievement-Literature review 1997-2015. J. Curric. Stud. 2017, 49, 703-721. [CrossRef]

34. Moher, D.; Shamseer, L.; Clarke, M.; Ghersi, D.; Liberati, A.; Petticrew, M.; Shekelle, P.; Stewart, L.A. Preferred reporting items for systematic review and meta-analysis protocols (prisma-p) 2015 statement. Syst. Rev. 2015, 4, 1. [CrossRef]

35. Moher, D.; Cook, D.J.; Eastwood, S.; Olkin, I.; Rennie, D.; Stroup, D.F. Improving the quality of reports of meta-analyses of randomised controlled trials: The quorom statement. Br. J. Surg. 2000, 87, 1448-1454. [CrossRef]

36. Álvarez-Bueno, C.; Pesce, C.; Cavero-Redondo, I.; Sánchez-López, M.; Garrido-Miguel, M.; Martínez-Vizcaíno, V. Academic achievement and physical activity: A meta-analysis. Pediatrics 2017, 140, e20171498. [CrossRef] [PubMed]

37. Bedard, C.; St John, L.; Bremer, E.; Graham, J.D.; Cairney, J. A systematic review and meta-analysis on the effects of physically active classrooms on educational and enjoyment outcomes in school age children. PLoS ONE 2019, 14, e0218633. [CrossRef]

38. Higgins, J.P.; Thompson, S.G.; Deeks, J.J.; Altman, D.G. Measuring inconsistency in meta-analyses. BMJ 2003, 327, 557-560. [CrossRef] [PubMed]

39. Higgins, J.P.; Altman, D.G.; Gøtzsche, P.C.; Jüni, P.; Moher, D.; Oxman, A.D.; Savovic, J.; Schulz, K.F.; Weeks, L.; Sterne, J.A. The Cochrane collaboration's tool for assessing risk of bias in randomised trials. BMJ 2011, 343, d5928. [CrossRef]

40. Higgins, J.P.T.; Thomas, J.; Chandler, J.; Cumpston, M.; Li, T.; Page, M.J.; Welch, V.A. Cochrane Handbook for Systematic Reviews of Interventions; John Wiley \& Sons: Chichester, UK, 2019.

41. Ardoy, D.N.; Fernández-Rodríguez, J.; Jiménez-Pavón, D.; Castillo, R.; Ruiz, J.; Ortega, F. A physical education trial improves adolescents' cognitive performance and academic achievement: The edufit study. Scand. J. Med. Sci. Sports 2014, 24 , e52-e61. [CrossRef]

42. García-Hermoso, A.; Hormazábal-Aguayo, I.; Fernández-Vergara, O.; González-Calderón, N.; Russell-Guzmán, J.; Vicencio-Rojas, F.; Chacana-Cañas, C.; Ramírez-Vélez, R. A before-school physical activity intervention to improve cognitive parameters in children: The active-start study. Scand. J. Med. Sci. Sports 2020, 30, 108-116. [CrossRef]

43. Mavilidi, M.F.; Lubans, D.R.; Eather, N.; Morgan, P.J.; Riley, N. Preliminary efficacy and feasibility of the "thinking while moving in English": A program with integrated physical activity into the primary school english lessons. Children 2018, 5, 109. [CrossRef]

44. Sallis, J.F.; Lewis, M.; McKenzie, T.L.; Kolody, B.; Marshall, S.; Rosengard, P. Effects of health-related physical education on academic achievement: Project spark. Res. Q. Exerc. Sport 1999, 70, 127-134. [CrossRef]

45. Beck, M.M.; Lind, R.R.; Geertsen, S.S.; Ritz, C.; Lundbye-Jensen, J.; Wienecke, J. Motor-enriched learning activities can improve mathematical performance in preadolescent children. Front. Hum. Neurosci. 2016, 10, 645. [CrossRef]

46. Davis, C.L.; Tomporowski, P.D.; McDowell, J.E.; Austin, B.P.; Miller, P.H.; Yanasak, N.E.; Allison, J.D.; Naglieri, J.A. Exercise improves executive function and achievement and alters brain activation in overweight children: A randomized, controlled trial. Health Psychol. 2011, 30, 91-98. [CrossRef] [PubMed]

47. Donnelly, J.E.; Hillman, C.H.; Greene, J.L.; Hansen, D.M.; Gibson, C.A.; Sullivan, D.K.; Poggio, J.; Mayo, M.S.; Lambourne, K.; Szabo-Reed, A.N.; et al. Physical activity and academic achievement across the curriculum: Results from a 3-year clusterrandomized trial. Prev. Med. 2017, 99, 140-145. [CrossRef] [PubMed]

48. Fakri, N.F.N.; Hashim, H.A. The effects of integrating physical activity into mathematic lessons on mathematic test performance, body mass index and short term memory among 10 year old children. J. Phys. Educ. Sport 2020, 20, 425-429.

49. Fedewa, A.L.; Ahn, S.; Erwin, H.; Davis, M.C. A randomized controlled design investigating the effects of classroom-based physical activity on children's fluid intelligence and achievement. Sch. Psychol. Int. 2015, 36, 135-153. [CrossRef]

50. Gao, Z.; Hannan, P.; Xiang, P.; Stodden, D.F.; Valdez, V.E. Video game-based exercise, latino children's physical health, and academic achievement. Am. J. Prev. Med. 2013, 44, S240-S246. [CrossRef]

51. Hraste, M.; De Giorgio, A.; Jelaska, P.M.; Padulo, J.; Granić, I. When mathematics meets physical activity in the school-aged child: The effect of an integrated motor and cognitive approach to learning geometry. PLoS ONE 2018, 13, e0196024. [CrossRef]

52. Layne, T.; Yli-Piipari, S.; Knox, T. Physical activity break program to improve elementary students' executive function and mathematics performance. Education 2021, 49, 583-591. [CrossRef]

53. Lubans, D.R.; Beauchamp, M.R.; Diallo, T.M.O.; Peralta, L.R.; Bennie, A.; White, R.L.; Owen, K.; Lonsdale, C. School physical activity intervention effect on adolescents' performance in mathematics. Med. Sci. Sports Exerc. 2018, 50, 2442-2450. [CrossRef]

54. Mavilidi, M.F.; Drew, R.; Morgan, P.J.; Lubans, D.R.; Schmidt, M.; Riley, N. Effects of different types of classroom physical activity breaks on children's on-task behaviour, academic achievement and cognition. Acta Paediatr. 2020, 109, 158-165. [CrossRef] 
55. Mavilidi, M.F.; Okely, A.; Chandler, P.; Louise Domazet, S.; Paas, F. Immediate and delayed effects of integrating physical activity into preschool children's learning of numeracy skills. J. Exp. Child. Psychol. 2018, 166, 502-519. [CrossRef]

56. Mavilidi, M.F.; Vazou, S. Classroom-based physical activity and math performance: Integrated physical activity or not? Acta Paediatr. 2021, 110, 2149-2156. [CrossRef] [PubMed]

57. Mullender-Wijnsma, M.J.; Hartman, E.; De Greeff, J.W.; Doolaard, S.; Bosker, R.J.; Visscher, C. Physically active math and language lessons improve academic achievement: A cluster randomized controlled trial. Pediatrics 2016, 137, e20152743. [CrossRef] [PubMed]

58. Polo-Recuero, B.; Moreno-Barrio, A.; Ordonez-Dios, A. Physically active lessons: Strategy to increase scholars' physical activity during school time. Rev. Int. Cien. Dep. 2020, 16, 342-357. [CrossRef]

59. Quinto Romani, A.; Klausen, T.B. Physical activity and school performance: Evidence from a danish randomised schoolintervention study. Scand. J. Educ. Res. 2017, 61, 479-502. [CrossRef]

60. Shoval, E.; Sharir, T.; Arnon, M.; Tenenbaum, G. The effect of integrating movement into the learning environment of kindergarten children on their academic achievements. Early Child. Educ. J. 2018, 46, 355-364. [CrossRef]

61. Tarp, J.; Domazet, S.L.; Froberg, K.; Hillman, C.H.; Andersen, L.B.; Bugge, A. Effectiveness of a school-based physical activity intervention on cognitive performance in danish adolescents: Locomotion-learning, cognition and motion-A cluster randomized controlled trial. PLoS ONE 2016, 11, e0158087. [CrossRef]

62. Vazou, S.; Skrade, M.A.B. Intervention integrating physical activity with math: Math performance, perceived competence, and need satisfaction. Int. J. Sport Exerc. Psychol. 2017, 15, 508-522. [CrossRef]

63. Watson, A.J.L.; Timperio, A.; Brown, H.; Hesketh, K.D. A pilot primary school active break program (acti-break): Effects on academic and physical activity outcomes for students in years 3 and 4. J. Sci. Med. Sport 2019, 22, 438-443. [CrossRef]

64. Resaland, G.K.; Aadland, E.; Moe, V.F.; Aadland, K.N.; Skrede, T.; Stavnsbo, M.; Suominen, L.; Steene-Johannessen, J.; Glosvik, Ø.; Andersen, J.R.; et al. Effects of physical activity on schoolchildren's academic performance: The active smarter kids (ask) cluster-randomized controlled trial. Prev. Med. 2016, 91, 322-328. [CrossRef]

65. Riley, N.; Lubans, D.R.; Holmes, K.; Morgan, P.J. Findings from the easy minds cluster randomized controlled trial: Evaluation of a physical activity integration program for mathematics in primary schools. J. Phys. Act. Health 2016, 13, 198-206. [CrossRef]

66. Ahamed, Y.; Macdonald, H.; Reed, K.; Naylor, P.J.; Liu-Ambrose, T.; McKay, H. School-based physical activity does not compromise children's academic performance. Med. Sci. Sports Exerc. 2007, 39, 371-376. [CrossRef] [PubMed]

67. Hagins, M.; Rundle, A. Yoga improves academic performance in urban high school students compared to physical education: A randomized controlled trial. Mind Brain Educ. 2016, 10, 105-116. [CrossRef]

68. Lima, R.A.; Soares, F.C.; Bezerra, J.; de Barros, M.V.G. Effects of a physical education intervention on academic performance: A cluster randomised controlled trial. Int. J. Environ. Res. Public Health 2020, 17, 4287. [CrossRef] [PubMed]

69. Mead, T.; Scibora, L.; Gardner, J.; Dunn, S. The impact of stability balls, activity breaks, and a sedentary classroom on standardized math scores. Phys. Educ. 2016, 73, 433. [CrossRef]

70. Shore, S.M.; Sachs, M.L.; DuCette, J.P.; Libonati, J.R. Step-count promotion through a school-based intervention. Clin. Nurs. Res. 2014, 23, 402-420. [CrossRef]

71. Pinto-Escalona, T.; Martinez-de-Quel, O. Ten minutes of interdisciplinary physical activity improve academic performance. Apunts Educ. Fís. Esports 2019, 138, 82-94. 